\title{
Asymmetric synthesis of secondary benzylic alcohols via arene chromium tricarbonyl complexes
}

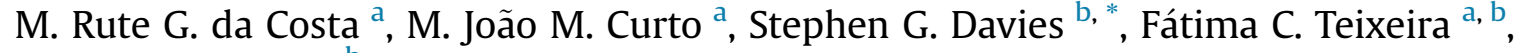 \\ James E. Thomson ${ }^{b}$ \\ a Laboratório Nacional de Energia e Geologia, I.P., Estrada do Paço do Lumiar, 22, 1649-038, Lisboa, Portugal \\ b Department of Chemistry, Chemistry Research Laboratory, University of Oxford, Mansfield Road, Oxford, OX1 3TA, UK
}

\section{A R T I C L E I N F O}

\section{Article history:}

Received 9 July 2018

Received in revised form

21 August 2018

Accepted 23 August 2018

Available online 25 August 2018

\section{Keywords:}

Arene chromium tricarbonyl

Secondary alcohols

Asymmetric synthesis

Hydroxylamines

\begin{abstract}
A B S T R A C T
(Aryl aldehyde)- and (aryl ketone)-chromium tricarbonyl complexes ortho-substituted with the chiral auxiliary $\mathrm{O}$-methyl- $\mathrm{N}$-( $\alpha$-methylbenzyl)hydroxylamine undergo diastereoselective addition of Grignard reagents and Super-Hydride ${ }^{\circledR}$, respectively, to give the corresponding secondary alcohols in high diastereoisomeric purity. These compounds may be easily decomplexed and deprotected to give the corresponding enantiopure amino alcohols.
\end{abstract}

๑) 2018 Elsevier Ltd. All rights reserved.

\section{Introduction}

Compounds bearing optically active secondary alcohols are an important group of molecules, being present in many natural products and biologically active compounds, and also as intermediates in the synthesis of other organic functionalities [1,2]. Over the years, there have been a number of studies on the synthesis of non-racemic secondary alcohols from achiral carbonyl compounds via asymmetric induction [3]. The two major methods for the enantioselective synthesis of non-racemic secondary alcohols are the enantioselective nucleophilic addition to aldehydes and the enantioselective reduction of unsymmetrical ketones $[1,3,4]$. In these cases, a chiral reducing agent or catalyst interacts with a prochiral substrate. Stereoselective nucleophilic additions to ortho-substituted (aryl aldehyde)- and (aryl ketone)-chromium tricarbonyl complexes have been achieved with achiral reducing agents, because the carbonyl group is already in a chiral environment. Usually, these nucleophilic additions occur with very high diastereoselectivities, as a result of attack on the carbonyl group from the uncomplexed face of the arene, since the chromium tricarbonyl unit sterically blocks the other face of the carbonyl group.

\footnotetext{
* Corresponding author.

E-mail address: steve.davies@chem.ox.ac.uk (S.G. Davies).
}

The conformation of the carbonyl, which could have the oxygen anti or syn to the ortho-substituent, can be predicted on the basis of known effects such as steric hindrance, dipolar repulsion or hydrogen bonding, leading to the preferred diastereoisomer upon nucleophilic addition [5]. We have recently reported the synthesis of (aryl aldehyde)- and (aryl ketone)-chromium tricarbonyl complexes ortho-substituted with the chiral auxiliary $O$-methyl- $N-(\alpha-$ methylbenzyl)hydroxylamine [6]. For example, enantiomerically pure complex $(1 p S, \alpha R)-4$ was prepared upon deprotonation of $(R)$ $O$-methyl- $N$ - $(\alpha$-methylbenzyl $)$ hydroxylamine with BuLi followed by addition of the resultant lithium amide to ( $\eta^{6}$-fluorobenzene) tricarbonylchromium $(0)$ 1, which gave $(R)-2$ in 56\% yield. Subsequently, a solution of $(R)-2$ in $\mathrm{Et}_{2} \mathrm{O}$ at $-78^{\circ} \mathrm{C}$ was treated with $t$-BuLi to effect diastereoselective ortho-deprotonation to give lithiated aryl anion 3, which was reacted with ethyl formate to give orthoformyl substituted complex $(1 p S, \alpha R)-\mathbf{4}$ in $82 \%$ yield as a single diastereoisomer (>99:1 dr) after chromatographic purification (Scheme 1). Unfortunately, reaction of lithiated aryl anion 3 with aldehydes gave relatively poor diastereoselectivity ( 60:40 dr) upon formation of the new benzylic stereogenic centre, and this low diasteroselectivity can be explained by the absence of steric or electronic control elements. We have observed similarly low diastereoselectivity $[5 f, g, j]$, when the anion derived from $(S)-[(\alpha-$ methylbenzyloxy)benzene]tricarbonylchromium( 0$)$ and the anion derived from [diphenyl sulfoxide]tricarbonylchromium $(0)$ were 